\title{
Féeries
}

Études sur le conte merveilleux, XVII $-\mathrm{XIX}{ }^{\mathrm{e}}$ siècle

$17 \mid 2021$

Contes en couleur

\section{Ombres et clartés dans les Mille et Une Nuits de} Galland

Shadows and Lights in The Thousand and One Nights Translated by Galland

Jean-Paul Sermain

\section{(2) OpenEdition}

1 Journals

Édition électronique

URL : https://journals.openedition.org/feeries/3624

DOI : $10.4000 /$ feeries.3624

ISSN : 1957-7753

Éditeur

UGA Éditions/Université Grenoble Alpes

Édition imprimée

ISBN : 978-2-37747-327-4

ISSN : 1766-2842

Référence électronique

Jean-Paul Sermain, «Ombres et clartés dans les Mille et Une Nuits de Galland », Féeries [En ligne], 17 |

2021, mis en ligne le 17 décembre 2021, consulté le 14 janvier 2023. URL : http://

journals.openedition.org/feeries/3624; DOI : https://doi.org/10.4000/feeries.3624

Ce document a été généré automatiquement le 14 janvier 2023.

Tous droits réservés 


\title{
Ombres et clartés dans les Mille et Une Nuits de Galland
}

\author{
Shadows and Lights in The Thousand and One Nights Translated by Galland
}

Jean-Paul Sermain

1 Les Mille et Une Nuits sont voisins, à la fois historiquement et littérairement, du Décaméron et de la tradition de la novella que ce recueil porte aux sommets, et cela aussi bien dans leur domaine, leur esprit, leur style. Cette tradition passée en France à la Renaissance s'est ensuite limitée et spécialisée dans les nouvelles historiques et galantes, dont le sujet amoureux s'accompagne d'une sorte de restriction dans ses objets et son style, jusqu'à l'assèchement. En mettant en français et en recomposant les Mille et Une Nuits à la fin du règne de Louis XIV ${ }^{1}$, Antoine Galland ramène l'esprit de la novella italienne et francisée, avec des matériaux largement inédits et hybridés de merveilleux, ce que la novella ne faisait qu'à petites doses (chez Boccace ou dans les Nuits facétieuses de Straparole). Ce qui pouvait passer pour un élargissement et un renouvellement du conte de fées consistait donc plus exactement à le rattacher à un registre du conte désormais ancien, et à renforcer son accueil du surnaturel et du fantastique, au sens de ce mot à l'époque, attesté dans les Mille et Une Nuits de Galland².

2 Le courant de la novella médiévale prolongé à la Renaissance et comme estompé par le $\mathrm{XVII}^{\mathrm{e}}$ siècle français revient ainsi par le biais d'une curiosité pour l'Orient et de l'entreprise originale de Galland, à la fois érudit et soucieux de faire partager son enthousiasme pour le Levant à un public d'honnêtes gens formés à la littérature que nous appelons classique. Ce courant restait en partie familier avec Cervantès et avec l'Arioste, ou les héritiers désinvoltes du picaresque comme Scarron et Lesage. Le cadre dramatique mis en place dans le premier récit du recueil et annoncé par le titre assurait le «tout ensemble » des contes et permettait d'apprécier l'étendue de leur matière comme la diversité de leurs registres, des situations, des points de vue, des motifs : l'œuvre dans sa totalité (certes plus réduite que dans les éditions arabes du $\mathrm{XIX}^{\mathrm{e}}$ siècle qui servent de support à nos modernes traductions) transportait le lecteur aux limites des terres connues et surtout l'amenait à apprécier l'intérêt pittoresque, moral et dramatique des éléments du monde sensible qui échappaient aux fictions 
contemporaines françaises presque entièrement orientées vers les langages de la bonne société. Ainsi une lampe ordinaire ou une jarre d'olive où cacher ses économies ou dissimuler des sbires commandaient d'amples histoires ou des développements cruciaux. Le rôle privilégié des voyageurs dans le recueil conduit à la découverte de palais somptueux ou de lieux terrifiants, à l'exploration d'îles et de palais, dont l'évocation fait partie de l'histoire.

Pour autant ces éléments matériels, propres à une géographie étendue et à des sociétés vues à tous les niveaux, restent subordonnés à la logique des contes et ne sont introduits que pour leur participation à une action, à des conflits, à des aventures. Le lecteur peut dès la première histoire du recueil imaginer son héros apercevoir dans la campagne un noyer et s'installer près d'une fontaine, puis manger des dattes et en jeter les noyaux. Mais si toutes ces choses sont mentionnées, elles gardent un caractère général, comme emblématique ; aucune description ne les singularise et elles servent de support à un événement extraordinaire : l'irruption d'un génie qui accuse le héros éberlué d'avoir tué son fils par un des noyaux de dattes et le condamne à mort. Quand, après un sursis d'un an, il revient se livrer au génie, trois vieillards qui surviennent, l'un accompagné d'une biche, l'autre de deux chiens noirs, le troisième simplement curieux, vont être invités à raconter leur histoire et le génie en récompense fait grâce au voyageur. Le texte n'arrête pas le lecteur sur le paysage ou ces animaux mais va apprendre les raisons et le sens de leur présence.

\section{Couleurs - l'indifférence du genre}

4 Très souvent sont mentionnés les vêtements luxueux ou les intérieurs exceptionnels mais le texte ne cherche ni à les faire voir, ni à les faire imaginer. Aussi restent-ils sans couleurs. Ce qui rend principalement ce monde étrange, nous dirions exotique, est ce qui s'y passe. C'est pourquoi l'une des très rares qualifications par la couleur de « l'eau jaune, couleur d'or » dans le dernier conte du recueil, « Les deux sœurs jalouses de leur cadette ", en suggère par analogie le prix et une étrangeté identique à celle de l'arbre qui chante ou de l'oiseau attirant ses semblables: ces merveilles sont à l'origine d'aventures mirobolantes qui vont conduire les trois héros à découvrir leur origine et à retrouver leur père. La couleur est comme arrachée au monde naturel et entre dans le surnaturel. Il en va de même dans l'histoire du pêcheur ${ }^{3}$ à qui sont échus successivement des poissons de quatre couleurs (blancs, rouges, bleus, jaunes): ils surprennent d'abord pour se carboniser dès qu'on tente de les cuire, puis ils se révèlent être les habitants du pays métamorphosés et distingués selon leur religion (musulmans, adorateurs du feu, chrétiens et juifs); ils ne valaient que par leur diversité qui symbolise celle des peuples et des croyances, et par leur commune esquive de toute cuisine. La couleur est transitoire : elle amorce la curiosité et n'a aucun lien propre avec ce qu'elle signifie finalement.

5 Comme l'herbe est peut-être verte (ou jaune?), la couleur est majoritairement impliquée par des matières et ce sont elles que retient le récit, presque toujours pour leur caractère précieux, et c'est par leur accumulation qu'elles méritent d'entrer dans le conte. Elles sont réservées au milieu des maîtres ou aux enchanteurs, et ont donc une place limitée. Dominent dans cet ensemble l'or et l'argent, puis viennent les diamants et les perles, suivis des rubis et des émeraudes. Ainsi l'idole d'or massif ${ }^{4}$ a des yeux de rubis et Aladdin fait incruster de pierres précieuses les vantaux des fenêtres dans la 
salle suprême de son palais. On peut leur associer les marbres, l'albâtre, l'onyx, le jaspe. À ces mentions récurrentes, on peut ajouter les exceptions d'un « étui de satin jaune et de soie verte » dont est sorti un instrument de musique ${ }^{5}$, un tapis de bouquets de roses de soie rouge ${ }^{6}$. Quant aux « couleurs vives » dont sont peintes les toiles dans ce conte, on ignorera quelles elles sont et seul le genre de ce qu'elles représentent est indiqué.

Ces pierres précieuses font partie d'un trésor qui magnifie le souverain ${ }^{7}$. Elles ornent aussi ses femmes et ses filles et en accentuent l'attrait. C'est ce qui en fait l'expression d'une splendeur hyperboliquement orientale telle que Galland en avait eu l'expérience frappante lors de son séjour à Constantinople et dans diverses parties de l'empire ottoman $^{8}$. Elles restent exceptionnelles dans l'univers du conte et pour l'essentiel à l'extérieur de l'action. L'histoire du Prince Ahmed est aussi fondée sur trois objets magiques qui donnent lieu à une longue quête, une pomme, une longue vue et un tapis, mais ils n'intéressent que par leur pouvoir magique : la première guérit, la seconde aide à voir à distance, le troisième vole. L'astuce du conte vient de ce que ces objets achetés pour surprendre vont servir conjointement à sauver la fille du souverain. C'est leur destin pratique qui surprend.

7 L'inspiration jugée aujourd'hui plus folklorique des scénarios dont s'empare Galland pour rédiger à peu près le dernier tiers de ses contes explique peut-être la fascination pour ces amoncellements de raretés. Ils s'étalent dans l'histoire d'Aladdin, dans celle du cheval enchanté, dans celle du Prince Ahmed, dans l'histoire des deux sœurs. Mais ce qui importe là encore, c'est ce qu'en font les héros. Ali Baba doit dissimuler la part du trésor qu'il a enlevé aux voleurs, pour ne pas attirer l'attention puis éviter les représailles. Ce que fait Aladdin des pierres précieuses, les ranger derrière un coussin, témoigne de son innocence et sa mère ne réveille le génie de la lampe que parce qu'elle a voulu la nettoyer. Dans le conte de Cogia Hassan Alhabbal, la famille veut garder le diamant surdimensionné trouvé dans un poisson que leur ami pêcheur leur remet par gratitude, uniquement parce qu'il met un peu de lumière dans une maison très sombre ou peut servir de jouet aux enfants.

Limités à des zones du récit assez restreintes, au monde d'en haut et au surnaturel, les objets de couleur restent subordonnés à leur intégration à la langue du conte, qui est celle des actions, des comportements, des décisions, des désirs, des passions. Aussi interviennent-ils dans la mesure où ils partagent une propriété plus générale, qui, elle, est fondamentale dans l'économie narrative, leur rayonnement et leur clarté. Ils entrent si l'on peut dire dans l'histoire parce qu'ils condensent ou reflètent la lumière, et par là dissipent les ténèbres, et c'est cette opposition qui est fondamentale dans l'œuvre.

\section{Lumières - la préférence de l'œuvre}

9 En effet, ces deux propriétés d'ombre et de clarté, peut-être à cause des conditions climatiques d'un Orient écrasé de soleil et cherchant à s'en protéger, entrent dans l'expérience des personnages, sont les conditions de leur action et même de leur vie, sont des repères et orientent leur désir, leur crainte ou leur attente. L'intégration de l'ombre et de la clarté à l'action se prête ainsi à une sorte d'intériorisation subjective. Elles deviennent l'expression d'une alternative entre la mort et la vie, le néant et le salut, souvent même le bien et le mal, le beau et le laid. Elles sont consubstantielles au sentiment du réel et prennent par là une dimension sensible. Dans le reflet, la clarté est 
comme un affleurement ; éclat, elle éblouit et pénètre. L'ombre enveloppe et submerge, étouffe et aveugle. Dans cette lutte entre le clair et l'obscur, intervient en tierce presque uniquement la couleur rouge avec ses développements dramatiques: le rougeoiement du feu et du sang qui coule se transforme cruellement en charbon et en nuit. L'émotion est à son comble et capte le lecteur.

Cette alternance scande l'action et ménage un accès choisi au monde oriental. Elle est amorcée dans le récit initial qui sert de cadre à l'ensemble des histoires et en fait, par sa tension propre et la situation exceptionnelle de contage ainsi établie, une unité organique. En effet, s'y trouvent bien des éléments ensuite développés et variés qui créent une atmosphère de clair-obscur chargée de drames pathétiques. Pour simplifier suivons l'ordre du récit. Ce qui engage l'action est, après que Schahzenan a entamé son voyage pour retrouver son frère, son retour sur ses pas pour dire un dernier adieu à sa femme. C'est la nuit mais, «à la clarté des flambeaux, qui ne s'éteignent jamais dans les appartements des princes et des princesses, il aperçut un homme dans ses bras ${ }^{9} »$. Cet amant est " un des derniers officiers de la maison». Schahzenan est alors pris d'» une affreuse mélancolie». L'éclairage a giorno est comme ignoré par les deux corps endormis et produit la nuit de l'humeur noire et de la rumination obsessionnelle - l'époux trompé revit dans son imagination la scène stupéfiante. Arrivé chez son frère Schariar, il fuit les divertissements préparés pour lui et, resté seul, il surprend à sa fenêtre qui donne sur le jardin vingt suivantes rejoindre dix noirs et s'accoupler (en binôme ?), tout comme le fait la sultane avec un autre noir perché sur un arbre et qu'elle appelle spécialement Massoud. La réunion se termine par un bain de minuit. Schahzenan se fait alors une raison de son infortune, mais son frère lui demande ce qui a chassé sa "noire mélancolie » et finit par avouer ce qu'il a vu, et tous les deux assistent à une nouvelle séance nocturne d'ébats amoureux. Ils se lèvent « dès le point du jour » et voient dans le désert une grosse colonne noire qui fend l'eau : en sort un génie tenant prisonnier une femme qui contraint les deux frères à l'honorer et se vante d'avoir trompé ainsi son mari pour la centième fois. Schariar décide alors d'enchaîner les mariages en ne gardant chaque femme qu'une seule nuit et en la faisant exécuter le lendemain matin. L'alternance nocturne et diurne prend une dimension terrifiante. Shéhérazade, la fille du vizir chargée d'appliquer l'économie des nuits voulue par le sultan, va la changer radicalement. Ayant imposé à son père son mariage avec le sultan, elle organise la nuit conjugale destinée à être unique en faisant installer sa sœur dans la chambre des époux, et en lui demandant de solliciter auprès d'elle un conte « une heure avant le jour». Elle commence son conte, mais dès qu'elle "s'aperç[oi]t qu'il [est] jour ", elle s'interrompt, laisse son mari vaquer à ses affaires, et « le lendemain, avant que le jour par[aisse] », sa sœur lui demande de poursuivre son récit. Ainsi s'engage une longue alternance de la nuit au jour qui se substitue à celle programmée criminellement par le sultan et qui devient le principe de sa guérison comme de la formation du recueil. C'est au terme de la nuit que le récit commence, et c'est « dès que le jour paraît qu'il s'interrompt " pour reprendre la nuit suivante. La nuit est donc ouverte aux autres mondes qui se déploient dans les histoires, elles-mêmes nécessaires pour que les conduites du jour suivent leur cours normal. Cette remémoration des contes a pour analogue dans l'histoire les sorties d'un autre sultan, lui devenu modèle mythique, Haroun-al-Raschid. Elles se font la nuit et sous couvert d'anonymat pour vérifier si l'empire est bien administré. Dans ses virées nocturnes, le sultan découvre les faits bouleversants qui suscitent la curiosité, et il la satisfait en imposant aux protagonistes de raconter leur histoire, et en conclusion il corrige les désordres ainsi 
révélés. En cela il relaie dans l'histoire de l'empire arabe le personnage de la conteuse : il profite de la nuit pour mettre à jour ce qui est caché, criminel, scandaleux, et en le faisant paraître et raconter il parvient à résoudre les mystères et à réparer les méfaits. Le recueil se construit sur la même tension entre l'ombre et la clarté que la conduite exemplaire du sultan héroïsé.

11 Dans l'ensemble du recueil élaboré par Galland, les rapports entre l'ombre et la lumière sont articulés sur deux niveaux. D'une part, ils se définissent par leur opposition matérielle et symbolique, le noir étant associé au bas, au mal, à la mort, et le clair à la beauté, au salut, au bonheur. D'autre part, ces conflits mêmes conduisent à leur transformation et ainsi sont pris dans une dialectique mobile. La valeur négative du noir vient d'abord des esclaves et des eunuques achetés ou razziés ${ }^{10}$. Noirs sont les amants de la sultane et de ses suivantes, noir l'amant indien de l'épouse du roi ${ }^{11}$, noir l'esclave qui s'est emparé de la pomme apportée par le mari à sa femme, et qui fait croire à ce dernier que celle-ci est infidèle, au point qu'il la massacre. Noir est aussi le lieu hostile: ainsi c'est dans un palais de marbre poli et d'acier qu'est enfermé et martyrisé le jeune roi. Dans les voyages de Sindbad, la montagne noire contient des aimants qui décomposent tous les bateaux. Noir est aussi souvent l'élément en quoi est transformé un personnage: il participe d'une dégradation magique. C'est le cas des deux chiens qui vont avec le voyageur de la première histoire; c'est le cas des deux chiennes dont s'occupe une des trois sœurs qui accueillent les calenders - ce sont deux sœurs jalouses ainsi punies. Dans l'histoire du pêcheur, comme je l'ai dit plus haut, les poissons délicieusement colorés deviennent, dès qu'on veut les cuire, du charbon. La consumation transforme l'être de chair en fumée puis en charbon: c'est le cas des époux incestueux découverts par le premier calender comme de l'héroïne persécutée par le génie - après l'épisode intermédiaire de la fournaise ${ }^{12}$. Le jeune roi est puni par sa femme enchanteresse qui transforme la partie inférieure de son corps en marbre noir. Dans la dernière histoire, le frère et la sœur partis en quête des objets merveilleux deviennent eux des pierres noires comme beaucoup d'imprudents avant eux. Sidi Nouman est enchanté par la beauté du visage de sa femme qu'il découvre le jour de son mariage ; mais il s'étonne qu'elle refuse de manger alors qu'elle est florissante. Il feint de dormir et la surprend qui court au cimetière déterrer avec une goule un cadavre et le dévorer. Après ce spectacle macabre, il lui fait des reproches et se voit transformé en chien. Il sombre dans l'horreur.

12 L'obscurité totale caractérise les lieux d'emprisonnement hermétiquement fermés. Dans l'histoire du pêcheur, c'est le cas pour le génie qui sort d'un vase de cuivre d'abord sur le mode d'une fumée et qui a souffert si longtemps qu'il veut tuer celui qui l'a délivré. Le pêcheur par ruse arrive à lui faire faire le chemin inverse, et le décide à redevenir fumée et à se glisser de nouveau dans le vase que le pêcheur s'empresse de refermer, avant de le délivrer une seconde fois. Lors de son quatrième voyage, Sindbad est jeté avec le cadavre de son épouse dans une vaste caverne où il doit s'attendre à mourir de faim. Ganem se retrouve la nuit dans un cimetière, la lumière qui apparaitt alors lui fait assister à un enterrement, et il découvre dans le coffre la femme qui y a été enfermée. Dans l'histoire du jeune roi déjà évoquée, le sultan voit un château de marbre noir et d'acier poli, dans lequel de grandes salles frappent au contraire par leur éclat lumineux avec leurs " étoffes des Indes relevées d'or et d'argent », et une fontaine dont l'eau jetée par un lion d'or retombe en perles et diamants après avoir presque touché un dôme peint d'arabesques. Cette combinaison des contraires caractérise aussi le héros d'abord signalé par des plaintes, puis remarquable par son corps hybride fait de 
chair en haut et de pierre noire en bas. Cette immobilisation monstrueuse permet à sa femme de fouetter jusqu'au sang ses épaules. Le lieu interdit où pénètre le troisième calender est voûté et éclairé de flambeaux d'or massif. Mais un cheval noir va lui faire perdre un œil.

Certaines noirceurs font partie de l'identité, comme celle de la race, de la carbonisation, de la pierre, elles sont naturellement irrémédiables et seuls des enchantements les annulent parfois. Mais l'obscurité absolue, l'enfermement appellent la clarté et la délivrance : elles scandent les étapes de l'histoire. Sindbad, jeté dans une caverne à côté du cadavre de sa femme défunte, survit en achevant les autres condamnés et en s'emparant de leur maigre provision. "Un jour", il devine une " espèce d'ombre " et il poursuit: " [...] j'aperçus enfin une lumière qui ressemblait à une étoile. Je marchai vite vers cette lumière, la perdant quelquefois selon les obstacles qui me la cachaient $[. . .]^{13}$.» Dans son sixième voyage, il échoue dans une île, au seuil d'une montagne qui regorge de pierres précieuses - encore un trésor à disposition des plus malins - et d'une source "d'une espèce de poix ou de bitume » qui devient de l'ambre : là encore le plus noir laisse la place au doré. La rivière ne débouche pas sur la mer mais s'engouffre dans la montagne. Le héros décide de s'y aventurer sur un radeau, il s'abandonne au courant "sans jamais apercevoir le moindre rayon de lumière ", puis il se réveille au grand jour et entouré de noirs, indigènes bienveillants par exception. Noureddin et la belle Persienne se sont introduits dans le pavillon d'été du sultan et ont fait allumer les bougies aux quatre-vingts fenêtres qui l'illuminent. Dans la nuit, c'est ce que surprend de loin le sultan à qui cette illumination puissante est réservée. Il rejoint alors les deux époux en fuite et se faufile à leurs côtés sous une fausse identité. Il ne les punit pas et admire avec eux «le jardin au clair de lune ${ }^{14}$ ». La vie d'Aladdin bascule quand il se cache pour surprendre la fille du sultan au bain et qu'en la voyant nue, il en tombe amoureux. La chair suscite en effet le désir en irradiant la lumière. La blancheur de neige s'oppose ainsi radicalement à la peau noire des esclaves et des eunuques. Le destin de Bedreddin Hassan change quand le génie est frappé par «l'éclat de sa beauté » capable de "mettre le monde en combustion ${ }^{15}$ ». Un tel éblouissement se reproduit quand le héros est transporté de la chambre amoureuse dans un cimetière: «Un petit vent qui commençait alors à souffler leva sa chemise, et laissa voir sa poitrine qui était plus blanche que la neige. Ils furent tous tellement étonnés de cette blancheur qu'ils firent un cri d'admiration [.... ${ }^{16}$.» Le tailleur de son côté se laisse envoûter par une femme à « la main plus blanche que l'albâtre ${ }^{17}$ ".

La femme enterrée vivante a un teint «frais et vermeil ${ }^{18}$ ». Le mot désigne une espèce de rouge " paillet " (Furetière, 1690), tirant sur le jaune, mais il signifie plutôt la bonne santé et est régulièrement associé à la fraîcheur. Le vieillard entièrement recouvert de poils blancs, une fois rasé montre un "teint vermeil ${ }^{19}$ ". Le rouge bascule dans l'autre sens quand le sang coule et que les blessures annoncent la mort. Dans la dernière histoire ${ }^{20}$, le couteau qui se couvre de sang annonce la mort du frère. Dans l'histoire d'Ali Baba, le chef des voleurs signale d'abord la maison du héros par une marque blanche, puis la seconde fois par une marque rouge; il enferme dans des jarres ses compagnons, qui y finissent ébouillantés par Morgiane qui le tuera dans une danse trompeuse. Comme le blanc signifie parfois l'annonce de la mort, le rouge est donc constamment ambivalent. Dans l'histoire de Beder, l'homme qui soulève le voile rouge rayé d'or de l'esclave est stupéfait de sa beauté. Le corps découpé de la femme crue infidèle est " plus blanc que de la neige ${ }^{21}$ » et il a été mis dans un paquet cousu d'un « fil rouge ». Aussi doit-on partir à la recherche de l'esclave noir qui est la cause 
(involontaire) de ce massacre. Le rouge est aussi celui de la braise ou du couteau mis dans le feu, c'est celui des yeux du monstre carnivore dans les voyages de Sindbad, et le tailleur se retrouve à la rue sans barbe et sans moustache avec les yeux rougis, c'est-àdire maquillés comme ceux d'une femme.

L'ombre et la clarté entrent ainsi dans la dynamique narrative des contes, dans leurs partages symboliques, dans le point de vue des personnages, et le lecteur y répond sur ces trois plans. Le titre du recueil des Mille et Une Nuits est très singulier car il ne mentionne ni un personnage ni un thème ni un événement, mais le moment où sont racontés les contes du recueil. Cette attention est renforcée par le caractère extraordinaire des conditions dans lesquelles ils sont produits, et par le sens éminemment dramatique et moral en même temps qu'elles leur confèrent. Le conte assure le passage de la nuit au jour en retenant sa menace meurtrière. Tout au long de l'œuvre vont s'opposer et s'imbriquer le jour et la nuit, et c'est par leur qualité sombre ou au contraire claire, leur assombrissement ou leur éclat que les différents acteurs, personnages ou choses, éléments naturels ou artificiels, se détachent et entrent dans des relations dramatiques, mais aussi sensibles et même affectives. Les couleurs assez rares, et surtout propres aux matériaux précieux, fascinent certes par leur caractère exceptionnel, mais elles n'interviennent dans le conte que par leur fonction dramatique, et valent finalement par leur intégration au système plus fondamental de l'ombre et de la clarté. L'opposition du corps noir et du corps blanc entre dans ce système et possède bien sûr une signification raciale : le blanc se purifie au sommet de la hiérarchie et les personnages les plus bas sont noirs. Mais outre que chacun est dialectiquement lié à l'autre et se modifie au cours de l'histoire, ils se rejoignent parfois par l'éclat, le brillant, le reflet. Au moment où s'achève la publication du recueil des Nuits de Galland, Étienne-Simon de Gamaches consacre au brillant toute la troisième partie de son ouvrage Les Agréments du langage réduits à leurs principes; mais, imbu de l'idéal classique de transparence au sens, Rousseau part au contraire en guerre contre cet effet trop recherché2 ${ }^{2}$. Toutefois, les œuvres littéraires maintiennent par leurs qualités propres les étapes anciennes de la sensibilité et du goût, prêtes à être réinvesties de nouveau. L'univers oriental devient avec les peintres romantiques un univers de couleurs; mais Fromentin est au contraire sensible à une sorte d'estompage par excès de luminosité, dans ses récits de voyage comme dans sa peinture : l'Orient y devient gris ou neutre. Il était déjà sans couleurs avec Galland, et déjà en clair-obscur, plus proche de Magnasco et de Piazzetta que de Pellegrini ou de Ricci.

\section{NOTES}

1. Voir A.Chraibi, Les Mille et Une Nuits. Histoire du texte et classification des contes, Paris, L'Harmattan, 2008 et Les Mille et Une Nuits, trad. A. Galland, présentation A. Chraibi et J.P. Sermain, Paris, GF Flammarion, 2004. Toutes les citations viennent de cette dernière édition.

2. T. II, p. 455.

3. T. I, p. 64 .

4. T. III, p. 273. 
5. T. I, p. 263.

6. T. II, p. 78.

7. Voir K. Pomian, Le Musée, une histoire mondiale, t. I : Du trésor au musée, Paris, Gallimard, coll. «Bibliothèque illustrée des histoires ", 2020, livre premier.

8. Voir A. Galland, Voyage à Constantinople, préface F. Bauden, Paris, Maisonneuve et Larose, 2002 (porte sur les années 1672-1673).

9. T. I, p. 24.

10. Voir O. Pétré-Grenouilleau, Les Traites négrières, Paris, Gallimard, 2004 et T. N’Diaye, Le Génocide voilé. Enquête historique, Paris, Gallimard, 2008.

11. T. I, p. 103.

12. Ibid., p. 162.

13. Ibid., p. 264.

14. T. II, p. 284-289.

15. T. I, p. 315.

16. Ibid., p. 325.

17. Ibid., p. 420.

18. T. II, p. 281.

19. T. III, p. 343.

20. Ibid., p. 347.

21. T. II, p. 393.

22. J. Berchtold, «Julie, ou le Contre Armide de Rousseau. Le procès du faux-brillant dans La Nouvelle Héloïse », dans A. Chamayou (éd.), La littérature et le brillant. Mélanges offerts à Pierre Malandain, Arras, Artois Presses Université. 2002, p. 105-138; « Le procès du faux brillant dans les Dialogues ", dans M. O’Dea (dir.), Jean-Jacques Rousseau en 2012. Puisque enfin mon nom doit vivre, Oxford, Voltaire Foundation, SVEC 2012:01, p. 45-62.

\section{RÉSUMÉS}

Les Mille et Une Nuits, telles qu'Antoine Galland les a mises en français et conçues, à partir d'un manuscrit excellent d'une partie de l'œuvre, peignent des mondes variés et curieux, mais n'accordent pas d'attention à ses couleurs, sauf si elles jouent un rôle dramatique (assez rarement) ou en lien avec le faste suprême des pierres précieuses et métaux rares. C'est un trait du conte. En revanche, le recueil est spécifiquement centré sur l'alternance du jour et de la nuit, et donne une grande place aux effets sensibles et bouleversants de l'ombre et de la clarté, et à leur interaction mobile.

The Thousand and One Nights, as translated and fashioned by Antoine Galland, from one of the best manuscript of the first part of the work, depict various worlds, but pay little attention to colours, except with dramatic meaning, or to suggest a world of luxury full of gems. This lack of colours is proper to tales. But the subject of The Thousand and One Nights is the recurrence of night and day, therefore gives a large part to the opposition of shadows and lights. 
INDEX

Mots-clés : Mille et Une Nuits, Antoine Galland, couleurs, ombres, clartés

Keywords : The Thousand and One Nights, Antoine Galland, colors, shadows, lights

\section{AUTEUR}

JEAN-PAUL SERMAIN

Université Sorbonne Nouvelle 